\title{
Risk Factors and Bronchopulmonary Dysplasia Severity. Data From the Spanish Bronchopulmonary Dysplasia Research Network
}

Cristina Ramos-Navarro ( $\nabla$ cramosn@salud.madrid.org )

Hospital General Universitario Gregorio Maranon https://orcid.org/0000-0002-6693-6405

Elena Maderuelo-Rodriguez

Hospital General Universitario Gregorio Marañón: Hospital General Universitario Gregorio Maranon

Ana Concheiro-Guisan

Alvaro Cunqueiro Hospital: Hospital Alvaro Cunqueiro

Santiago Perez-Tarazona

Hospital La Fe: Hospital Universitari i Politecnic La Fe

Santiago Rueda-Esteban

Hospital Clinico Universitario San Carlos

Ana Sánchez-Torres

Hospital La Paz: Hospital Universitario La Paz

Manuel Sánchez-Solis

Hospital Virgen de la Arrixaca: Hospital Clinico Universitario Virgen de la Arrixaca

Ester Sanz-López

Hospital General Universitario Gregorio Marañón: Hospital General Universitario Gregorio Maranon

Manuel Sánchez-Luna

Hospital General Universitario Gregorio Marañón: Hospital General Universitario Gregorio Maranon

\section{Research Article}

Keywords: GEIDIS, patients diagnosed with BPD, mechanical ventilation (MV)

Posted Date: May 27th, 2021

DOI: https://doi.org/10.21203/rs.3.rs-532632/v1

License: (1) (i) This work is licensed under a Creative Commons Attribution 4.0 International License.

Read Full License

Version of Record: A version of this preprint was published at European Journal of Pediatrics on October 1st, 2021. See the published version at https://doi.org/10.1007/s00431-021-04248-z. 


\section{Abstract}

GEIDIS is a national based research-net registry of patients with bronchopulmonary dysplasia (BPD) from public and private Spanish hospitals. It was created to provide data on the clinical characterization and follow up of infants with BPD until adulthood. The purpose of this observational study was to analyze the characteristics and the impact of perinatal risk factors on BPD severity. The study included 1,780 patients diagnosed with BPD. Of the total sample, $98.6 \%$ were premature (less than 37 weeks) and $89,4 \%$ less than 30 weeks of gestation. The median gestational age was 27.1 weeks $(25.8-28.5)$ and median birth weight $890 \mathrm{~g}(740-1,090 \mathrm{~g}) .52 .3 \%(\mathrm{n}=931)$ were classified as mild (type 1$), 25.1 \%(\mathrm{n}=447)$ were moderate (type 2), and $22.6 \%(n=402)$ severe BPD (type 3 ).

Most pre-and postnatal risk factors for type 2/3 BPD were associated with the length of exposure to mechanical ventilation (MV). Independent prenatal risk factors were male gender, oligohydramnios, and intrauterine growth restriction. Postnatal risk factors included the need for $\mathrm{FiO}_{2}$ of $>0.30$ in the delivery room, two or more doses of surfactant administration, nosocomial pneumonia, and the length of exposure to MV.

Conclusions: In this national based research-net registry of BPD patients the length of MV is the most important risk factor associated with type 2/3 BPD. Among type 3 BPD patients, those who required an $\mathrm{FiO}_{2}>.30$ at 36 weeks' postmenstrual age had a higher morbidity, during hospitalization and at discharge, compared to those with nasal positive pressure but $\mathrm{FiO}_{2}<.30$.

\section{Introduction}

Bronchopulmonary dysplasia (BPD) is the most frequent sequelae of prematurity and results in significant long-term health consequences and high healthcare costs [1-3]. It is characterized by impaired development of the alveoli, airways, and pulmonary vasculature, which is often associated with structural changes of the airways and vasculature. The main risk factor is immaturity, and the incidence increases as gestational age decreases. As a multifactorial disease, many external factors are involved in its severity such as, invasive mechanical ventilation-induced lung injury, oxidative stress, infections, inflammation, pulmonary overflow, nutritional disorders, and others that can affect the lungs' normal growth at early stages of their development.

A better understanding of factors linked to lung damage have led to changes in the management of very immature newborns, as controlling oxygen therapy, noninvasive respiratory support, early and less invasive surfactant therapy, prophylactic caffeine, infection prevention, and nutrition improvement among others, minimizing lung damage and promoting a better lung growth and development [1].

The incidence of BPD has not declined over the last decade [2], possibly because of an increase in the survival rate of the most premature infants who have a greater risk of developing BPD. Simultaneously, there has been a decrease in the severity of BPD [3]. 
The Spanish Bronchopulmonary Dysplasia Research Group (GEIDIS) is a national research network with a prospective online case registry of patients diagnosed with BPD from 66 Spanish hospitals created to better understand risk factors and long-term consequences until adulthood.

The purpose of this initial study was to define and analyze the characteristics of the BPD patients in the GEIDIS registry and describe the impact of pre- and postnatal factors on the severity of the disease.

\section{Methods.}

The study involves the analysis of the data collected prospectively in the GEIDIS registry between January 2016 and August 2020.

Inclusion criteria: Patients diagnosed with BPD (more than 28 days on respiratory support or an $\mathrm{FiO}_{2}>.21$ evaluated at 36 weeks postmenstrual age [PMA]) who had been entered in the GEIDIS registry database [4].

Definitions of the variables used in the study (specified in the database and described in Supplementary Information 1:

- Perinatal variables: Gestational age (GA); Sex; Birth weight (grams); Maternal age (years); Antenatal corticosteroids; Intrauterine growth restriction (IUGR); Oligohydramnios; Clinical and Histological chorioamnionitis; Type of respiratory support received at birth, during hospitalization, and at 36 weeks' PMA; Need for treatment with surfactant, inhaled nitric oxide therapy (iNO), postnatal corticosteroids therapy, diuretics, caffeine, and bronchodilators during hospitalization. Length of exposure to invasive mechanical ventilation (MV) in days. Respiratory support and treatments at discharge. Patent ductus arteriosus (PDA); Nosocomial sepsis; Nosocomial pneumonia; Air leak Syndrome (pneumothorax or interstitial emphysema); Pulmonary hypertension ; Necrotizing Enterocolitis requiring surgery (NEC); Intraventricular hemorrhage; Hemorrhagic stroke; Periventricular leukomalacia; Hydrocephalus requiring a shunt; Retinopathy of prematurity.

- $B P D$ was graded according to the agreed definition [4] into type 1 BPD (mild), type 2 BPD (moderate) and type $3 \mathrm{BPD}$ (severe). Patients with type $3 \mathrm{BPD}$ who need a $\mathrm{FiO}_{2}>.30$ were compared to patients who received positive-pressure respiratory support but at an $\mathrm{FiO}_{2}<.30$ at 36 weeks' PMA. Those on high-flow nasal cannula (more than $2 \mathrm{~L} / \mathrm{min}$ ) at 36 weeks' PMA were classified as type 3 BPD.

Statistical analysis:

The statistical data analysis was carried out with the aid of the IBM SPSS software package for Mac, version 21 (Chicago, Illinois).

The descriptive data analysis used measures of central tendency and measures of dispersion to summarize the quantitative variables and percentage distribution in the case of qualitative variables. 
We used the Chi-squared test $\left(\chi^{2}\right)$ for the comparative analysis of categorical variables with the Bonferroni correction for multiple comparisons, and the Kruskal-Wallis test with Bonferroni adjustment for the quantitative variables, as they did not meet the normality criteria. Statistical significance was set to a p-value $<0.05$.

Binary logistic regression was used for the multivariate analysis of binary response variables and linear regression for quantitative variables. The variables analyzed were selected according to their theoretical relevance and the level of significance observed in the bivariate analysis. The number of variables included in each analysis was determined based on the number of events/nonevents for the response variable. Given its clinical relevance, GA was included in all models. The approximate models included the interactions of the main study variable with the rest of the variables included, proceeding to stratification in the case of significant interactions.

The study was assessed and approved by the Local Research Ethics Committees and authorized by the Spanish Agency for Medicines and Medical Devices (AEMPS). Informed consent was obtained from the legal guardians of participants included in the study.

\section{Results}

The total sample included 1,780 patients with a diagnosis of BPD, 931 (52.3\%) with type 1 BPD, 447 (25.1\%) with type 2 BPD, and 402 (22.6\%) with type 3 BPD. The respiratory support needed by patients with type 3 BPD is detailed in Supplementary Table 1.

$98.6 \%(1,755 / 1,780)$ were less than 37 weeks' gestation and $89.4 \%(1,591 / 1,780)$ less than 30 weeks of gestation. The median GA was 27.1 weeks (25.8-28.5), and the median birth weight was $890 \mathrm{~g} \mathrm{(740-}$ $1,090 \mathrm{~g}) .25$ were born at term, of them 11 were diagnosed of congenital diaphragmatic hernia, 2 of bronchopneumonia, 2 of meconium aspiration syndrome, 5 of congenital heart disease, and 5 patients had other conditions.

The proportion of type 2/3 BPD decreased as GA increased until 30 weeks of gestation, over this gestational age the frequency of type 2/3 BPD increased (Fig. 1).

Patients with type 2/3 BPD had a higher rate of respiratory morbidity from birth and required respiratory support and oxygen for a longer time. They were also associated with a greater morbidity during hospitalization (Table 1), and at discharge (Table 2), especially for those with type 3 BPD.

All of the risk factors analyzed were also associated with a greater length of exposure to MV (Supplementary Table 2); only histological chorioamnionitis and the administration of caffeine seemed to be linked to less exposure to MV when adjusted for GA. However, the percentages of patients who needed MV was very similar for those with and without histological chorioamnionitis $(74.6 \%$ and $75.9 \%$, respectively, $\mathrm{p}$ 0.688), as was the length of exposure to $\mathrm{MV}$, median of 10 days for both patients with (3$22)$ and without histological chorioamnionitis $(3-25)(p=0.605)$. 
Median duration of MV was 5 hours $(0-18)$ in patients treated with caffeine $(n=1,732)$ and 26 hours $(0-$ 50) $(p=0.001)$ in those who were not $(n=37) .73 \%$ of patients who did not receive caffeine were born at $>30$ weeks of gestation.

When the estimation of the effect of the independent variables was adjusted for the length of exposure to $\mathrm{MV}$, sex (male), IUGR, oligohydramnios, need for $\mathrm{FiO}_{2}>.30$ at birth, two or more doses of surfactant, and nosocomial pneumonia were associated with a higher incidence of type 2/3 BPD. All the other variables, including GA, were not associated with a significant effect on type 2/3 BPD (Table 3).

In the group of patients with type $3 \mathrm{BPD}$, those who required an $\mathrm{FiO}_{2}>.30$ at 36 weeks' PMA had a higher morbidity, both during hospitalization and at discharge, compared to the group of patients with nasal positive pressure but at a $\mathrm{FiO}_{2}<.30$ (Supplementary Table 3). The was an increased incidence of oligohydramnios in patients who required $>30 \%$ oxygen at 36 weeks' PMA compared to those who needed positive-pressure ventilation, which was significant when adjusted for the rest of the prenatal risk factors (OR 2.07; 95\% Cl, 1.18-3.62).

Surgical NEC was associated with a reduced incidence of type 2/3 BPD when adjusted for the length of MV (Table 3). Each day on MV in patients with NEC was associated with an increased incidence of type 3 BPD of at least $1.1 \%(\mathrm{OR} 1.037 ; 95 \% \mathrm{Cl}, 1.011-1.063)$ compared to $6.1 \%$ in the other patients (OR 1.072; $95 \% \mathrm{Cl}, 1.061-1.084)$.

For patients who did not require MV ( $n=430 ; 24.2 \%$ of the sample), prenatal factors (male, IUGR, oligohydramnios) were associated with a greater incidence of type 2/3 BPD, but none of the postnatal factors presented a significant association. A higher GA was associated with a greater incidence of type 2/3 BPD in this group (Supplementary Table 4), although this association was no longer significant when only patients born at under 30 weeks' gestation are considered $(n=395)$ (OR 1.104; 95\% Cl, 0.890-1.368). In this group, $71.9 \%(n=309)$ developed type 1 BPD, 17.9\% $(n=77)$ type 2 BPD, and 10.2\% $(n=44$, of whom only six patients required $>30 \%$ oxygen at 36 weeks' PMA) were type 3 BPD. This is in contrast to patients who did require MV, where type 1 BPD was $46.2 \%$ ( $n=620)$, type 2 BPD $27.3 \%(n=366)$, and type 3 BPD 26.5\% $(n=356)(p<0.001)$.

When stratified according to GA, patients born $>30$ weeks' gestation had a higher proportion of males, IUGR, more cases of pneumothorax, pulmonary hypertension, iNO therapy, longer exposure to MV, and a similar percentage of type 2/3 BPD to infants born at less than 26 weeks (Table 4).

\section{Discussion}

This study confirms the important role of immaturity as a predisposing factor in the development of BPD. In addition, exposure to MV seems to act as an intermediary agent between risk factors and the severity of BPD. It is hard to establish the individual influence of each of the known risk factors on the severity of BPD, as they are all associated with exposure to MV. Evidence derived from both animal studies and randomized clinical trials has shown that MV is the most important factor associated to the development 
of BPD [5-8]. In this observational study, MV also acts as a variable in the causal pathway between most of the pre-and postnatal risk factors and the development of type 2/3 BPD.

Most of the risk factors studied were associated with a longer exposure to MV; only higher GA, chorioamnionitis, and caffeine therapy were protective factors.

GA is the strongest predictor of the development of BPD [2,9-11], which is also related to MV [12,13]. Immaturity, both pulmonary and neurological, results in a more severe respiratory failure, more need for surfactant therapy, central apnea, and morbidity during hospitalization [10], which means more exposure to MV. In our study the effect of GA on the severity of BPD was no longer significant when adjusted for the length of exposure to MV.

It should be noted that the effect of GA in our sample may be partly conditioned by the diagnostic criteria for BPD, which currently generate controversy [14-17].

The current classification of BPD lies mostly on the respiratory support required. It is defined BPD if needing more than 28 days oxygen therapy at a PMA of 36 weeks and grading the severity of BPD based on the respiratory support required [4]. One of the most controversial points is the diagnosis of type 1 BPD, patients who do not require respiratory support at 36 weeks' PMA but need oxygen for 28 days, as some authors believe that this can be attributed to immaturity [18] and does not imply the development of chronic lung disease [19]. In fact, some proposals for the disease's diagnosis and classification exclude the concept of type 1 BPD $[20,21]$. In our population we observed a higher proportion of type 1 BPD in neonates $<30$ weeks, in fact, most patients with BPD $(89,4 \%)$ were in this GA group. Also, the need for more than 28 days of oxygen or respiratory support selects a small and probably unrepresentative sample of the whole population of preterm infants born after 30 weeks, as they have higher morbidity (pneumothorax and pulmonary hypertension) and higher type 2/3 BPD proportion than more immature premature infants (26-30 weeks of gestation). This may interfere in the analysis of the impact of GA on BPD severity in our sample.

Jensen et al. analyzed the predictive power of 18 BPD classifications with respect to long-term respiratory morbidity and reported that the better predictive value for a classification was based solely on respiratory support, without including oxygen requirements at 36 weeks' PMA [21]. In our sample, a requirement of > $.30 \mathrm{FiO} 2$ at 36 weeks' PMA was associated with increased morbidity during hospitalization compared to the need for positive pressure support. The need for more supplemental oxygen is considered a better marker of both alveolar and vascular parenchymal lung disease than the need for positive-pressure therapy, which may be due to other causes, such as large airway diseases or central apnea [22]. However, it is also true that many patients often require an oxygen concentration above $30 \%$ with a low-flow nasal cannula due to atelectasis or airway collapse which can be reversed with positive-pressure treatment, and consequently the oxygen supply can be reduced. We are dealing, therefore, with an extremely complex disease with different phenotypes that appear to overlap in most cases [23]. 
Our series differs from others as it includes a low proportion of intubated patients at 36 weeks' PMA, only $4.8 \%$ compared to $13 \%$ in the United States series analyzed by Jensen et al. [21], a greater use of noninvasive positive pressure ( $40.4 \%$ vs $32.4 \%$ ), and a very similar percentage of patients with low-flow nasal cannula ( $54.7 \%$ vs $54.5 \%$ ). It also features relatively low percentages of patients with oxygen requirements above $30 \% ; 16 \%$ of the patients who needed respiratory support at 36 weeks and $33.6 \%$ when considering patients with type 3 BPD, compared to $46.7 \%$ and $87 \%$, respectively, in the Prematurity and Respiratory Outcomes Program cohort [24].

Given the above, we analyzed all the patients with type 3 BPD together (i.e., those requiring oxygen at > $30 \%$ combined with patients in need of positive-pressure respiratory support), although we do believe that an analysis of the differences between each type of respiratory support received at 36 weeks' PMA is very important when assessing long-term follow-up.

We found that chorioamnionitis was associated with a reduced need of MV after adjusting for GA. However, the percent of patients requiring MV and its duration were very similar for newborns with and without chorioamnionitis. The fact that chorioamnionitis was diagnosed more frequently among the most immature patients, and consequently those with a greater exposure to $\mathrm{MV}$, means that the adjustment for GA might be distorting the true impact of chorioamnionitis, which remains a causal factor for preterm birth. Other authors have also reported that the effect of chorioamnionitis is dependent on GA $[25,26]$. Pietrasanta et al. went so far as to question the suitability of adjusting the effect of chorioamnionitis for GA [26]. In this study, if chorioamnionitis is not adjusted for GA it does not correlate with the need for intubation at birth or the incidence of more severe forms of BPD.

Caffeine was associated with less exposure to MV, even when adjusted for GA. This effect should be interpreted with caution since there were very few patients who did not receive caffeine and most of them were born $>30$ weeks, which, as we have already seen, was a group of increased risk in this sample. This is consistent with data published in the literature, as the beneficial effect of caffeine decreasing the incidence of BPD is in part related to the reduced need for MV, mostly by reducing apnea $[27,28]$.

Male gender, oligohydramnios, IUGR, a requirement for FiO2 $>0.30$ at birth, more than one dose of surfactant, and nosocomial pneumonia were independently associated with the development of type $2 / 3$ BPD versus type 1 .

The association between male sex and an increased severity of BPD has already been reported by others[29,30]. In neonates born at under 32 weeks' gestation, the lungs of males are less mature than of females at the same GA, which suggests a sex-dependent differences in the canalicular and saccular phases of lung development [31,32]. In function of sex, different genetic patterns have also been described in mesenchymal cells taken from tracheal aspirates [33]. In our study, being male correlated with a greater exposure to MV, but its effect on the development of type 2/3 BPD was still significant after adjusting for this variable. Furthermore, in patients who were not exposed to MV, being male was also associated with a greater incidence of severe forms of BPD; that is, there were sex-dependent differences in lung development that were independent of a higher rate of initial respiratory morbidity. 
The effects of oligohydramnios and intrauterine growth restriction are also independent of exposure to MV. Oligohydramnios restricts intrauterine fetal lung growth, which causes pulmonary hypoplasia [34-36]. In our study sample, oligohydramnios is associated to a higher proportion of patients requiring $>30 \%$ oxygen at 36 weeks' PMA. This effect is consistent with the findings of other series in which oligohydramnios was associated with parenchymal pulmonary compromise, pulmonary vascular alterations [37-39] and pulmonary hypertension [40]. The correlation between impaired pulmonary vascular development and the emergence of BPD is widely discussed in the literature [41], with some authors suggesting it is one of the etiopathogenic mechanisms behind BPD (vascular theory of BPD) in which the impaired development of pulmonary vessels would dictate any alteration in distal airspace growth $[42,43]$.

The effect of intrauterine growth restriction (IUGR) on the development of BPD, seems to be related also to an inhibition of lung growth involving altered angiogenesis. Alterations in placental vascularization and perfusion observed in premature patients with IUGR appear to affect both lung development and vascular growth, resulting in a greater susceptibility to develop BPD and pulmonary hypertension $[44,45]$.

In our sample we also observed a relationship between an increased need for respiratory support at birth and the development of type 2/3 BPD. This pattern of severe respiratory failure from birth is the most frequently related to BPD development in other series [46]. The correlation between a greater need for respiratory support at birth and the appearance of type 2/3 BPD in our series occurred independently of the length of the subsequent exposure to $\mathrm{MV}$, which indicates pulmonary involvement from birth that predisposes the development of more severe forms of BPD.

Another interesting feature is the association between a diagnosis of NEC and a lower incidence of both type 2/3 BPD after adjusting for the length of exposure to MV. It is worth noting that since these patients underwent surgery, they were all exposed to MV. On the other hand, this exposure was due to their abdominal process that in many cases causes the need for sedation, rather than a respiratory disease, so usually this group of patients requires a lower level of ventilatory assistance than those patients who had respiratory failure. In fact, MV had a lesser effect on the development of type 3 BPD in patients with surgical enterocolitis than in the rest of the sample. This highlights the complexity of the mechanisms involved in lung damage and the difficulty of assessing their effect outside the controlled environment of animal studies. Nowadays, minimizing exposure to MV is an objective in all neonatal units, so its use is restricted to patients with severe respiratory failure when noninvasive respiratory support fails. The deleterious effect of $\mathrm{MV}$ is possibly greater in these patients who have lung disease and, therefore, an increased need for respiratory support and oxygen; but what is more, the necessity for MV leads us to select a group of patients with a higher risk of developing severe forms of BPD.

In patients who did not require MV during hospitalization, only being male and oligohydramnios were associated with a higher likelihood of developing type 2/3 BPD; however, none of the postnatal factors correlated with the emergence of type 2/3 BPD, which again emphasizes the role of MV as a mediator between these factors and the onset of the gravest forms of BPD. 
This study has some limitations as we only included patients with a diagnosis of BPD and given the observational character of the study, our assessment of the effect of the risk factors may be subject to significant biases, as the analysis only indicates the relationship between the risk factors and severity of BPD, as opposed to their effect on its incidence. Also, mortality in the first 28 days of life has not been considered. On the other hand, the large sample size provides a very representative description of the Spanish population with BPD, even though not all of the neonatal units attending preterm infants have participated.

In conclusion, in our national based research-net registry of patients with bronchopulmonary dysplasia, prenatal risk factors for the development of type 2/3 BPD versus type 1 BPD were male sex, IUGR and oligohydramnios. Exposure to $\mathrm{MV}$ is the main postnatal risk factor associated with the development of type 2-3 BPD, which also acts as an intermediary factor between all the other postnatal risk factors and the development of more severe types of BPD. Among type 3 BPD patients, those who required an $\mathrm{FiO}_{2}>.30$ at 36 weeks' PMA had a higher morbidity, during hospitalization and at discharge, compared to those with nasal positive pressure but with $\mathrm{FiO}_{2}<.30$.

\section{Abbreviations}

BPD Bronchopulmonary dysplasia

EN. Enteral nutrition

GA Gestational age

GEIDIS Spanish Bronchopulmonary Dysplasia Research Group

iNO inhaled nitric oxide therapy

IUGR Intrauterine growth restriction

MV Mechanical Ventilation

NEC Necrotizing Enterocolitis

PDA Paten Ductus arteriosus

PMA Postmenstrual age

\section{Declarations}

Acknowledgments: The authors would like to thanks Professor Eduardo Bancalari for assistance in revision the manuscript and the Institute for the Improvement in Healthcare (IMAS) for its support.

Funding: No funding was received for conducting this study 
Conflicts of interest: None of the authors declare commercial or financial involvement that might present a conflict of interest in connection with this manuscript.

Availability of data and material: Data are available on the online GEIDIS registry platform.

Code availability: N/A

Authors' contributions: Cristina Ramos Navarro designed the study, performed the acquisition and analysis of the data and draft the test. Elena Maderuelo-Rodríguez, Ana Concheiro-Guisán , Santiago Pérez-Tarazona, Santiago Rueda-Esteban, Ana Sánchez-Torres, Manuel Sánchez-Solís, Ester Sanz-López designed the study, collected data during the study period and revised the draft. Manuel Sánchez Luna designed the study, made contributions on the conception of the work and revised the draft critically.

Ethics approval: This article does not contain any interventional studies with human participants or animals performed by any of the authors. This study was approved and monitored by the Clinical Research Ethics Committee at Virgen de la Arrixaca Hospital. The study was performed in accordance with the ethical standards as laid down in the 1964 Declaration of Helsinki.

Consent to participate: Informed consent was obtained from the legal guardians of participants included in the study.

Consent for publication: All the authors revised and approved the final version of the text and consents its publication.

\section{References}

1. Owen LS, Manley BJ, Davis PG, et al. The evolution of modern respiratory care for preterm infants. Lancet. 2017 04;389(10079):1649-1659.

2. Stoll BJ, Hansen NI, Bell EF, et al. Trends in Care Practices, Morbidity, and Mortality of Extremely Preterm Neonates, 1993-2012. Jama. 2015 Sep 8;314(10):1039-51.

3. Abman SH, Bancalari E, Jobe A. The Evolution of Bronchopulmonary Dysplasia after 50 Years. Am J Respir Crit Care Med. 2017 02;195(4):421-424.

4. Jobe AH, Bancalari E. Bronchopulmonary dysplasia. Am J Respir Crit Care Med. 2001 Jun;163(7):1723-9.

5. Hernandez LA, Peevy KJ, Moise AA, et al. Chest wall restriction limits high airway pressure-induced lung injury in young rabbits. J Appl Physiol (1985). 1989 May;66(5):2364-8.

6. Jobe AH. Animal Models, Learning Lessons to Prevent and Treat Neonatal Chronic Lung Disease. Front Med (Lausanne). 2015;2:49.

7. Philip AG. Oxygen plus pressure plus time: the etiology of bronchopulmonary dysplasia. Pediatrics. 1975 Jan;55(1):44-50. 
8. Dreyfuss D, Soler P, Basset G, et al. High inflation pressure pulmonary edema. Respective effects of high airway pressure, high tidal volume, and positive end-expiratory pressure. Am Rev Respir Dis. 1988 May;137(5):1159-64.

9. Isayama T, Lee SK, Mori R, et al. Comparison of mortality and morbidity of very low birth weight infants between Canada and Japan. Pediatrics. 2012 Oct;130(4):e957-65.

10. Stoll BJ, Hansen NI, Bell EF, et al. Neonatal outcomes of extremely preterm infants from the NICHD Neonatal Research Network. Pediatrics. 2010 Sep;126(3):443-56.

11. Klinger G, Sokolover N, Boyko V, et al. Perinatal risk factors for bronchopulmonary dysplasia in a national cohort of very-low-birthweight infants. Am J Obstet Gynecol. 2013 Feb;208(2):115.e1-9.

12. Dargaville PA, Gerber A, Johansson S, et al. Incidence and Outcome of CPAP Failure in Preterm Infants. Pediatrics. 2016 07;138(1).

13. Fuchs $H$, Lindner W, Leiprecht $A$, et al. Predictors of early nasal CPAP failure and effects of various intubation criteria on the rate of mechanical ventilation in preterm infants of $<29$ weeks gestational age. Arch Dis Child Fetal Neonatal Ed. 2011 Sep;96(5):F343-7.

14. Bancalari E, Jain D. Bronchopulmonary Dysplasia: Can We Agree on a Definition? Am J Perinatol. 2018 May;35(6):537-540.

15. Hines D, Modi N, Lee SK, et al. Scoping review shows wide variation in the definitions of bronchopulmonary dysplasia in preterm infants and calls for a consensus. Acta Paediatr. 2017 Mar;106(3):366-374.

16. Ibrahim J, Bhandari V. The definition of bronchopulmonary dysplasia: an evolving dilemma. Pediatr Res. 2018 11;84(5):586-588.

17. Jobe AH, Steinhorn R. Can We Define Bronchopulmonary Dysplasia? J Pediatr. 2017 09;188:19-23.

18. Kinsella JP, Greenough A, Abman SH. Bronchopulmonary dysplasia. Lancet. 2006 Apr;367(9520):1421-31.

19. Ronkainen E, Dunder T, Peltoniemi O, et al. New BPD predicts lung function at school age: Follow-up study and meta-analysis. Pediatr Pulmonol. 2015 Jan.

20. Higgins RD, Jobe AH, Koso-Thomas M, et al. Bronchopulmonary Dysplasia: Executive Summary of a Workshop. J Pediatr. 2018 Mar 16.

21. Jensen EA, Dysart K, Gantz MG, et al. The Diagnosis of Bronchopulmonary Dysplasia in Very Preterm Infants: An Evidence-Based Approach. Am J Respir Crit Care Med. 2019 Apr 17.

22. Bancalari E, Claure N, Jain D. Diagnostic Classification of Bronchopulmonary Dysplasia: A Compromise between Defining Lung Disease versus Long-Term Outcome Prediction. Am J Respir Crit Care Med. 2019 11;200(10):1322-1323.

23. Wu KY, Jensen EA, White AM, et al. Characterization of Disease Phenotype in Very Preterm Infants with Severe Bronchopulmonary Dysplasia. Am J Respir Crit Care Med. 2020 06;201(11):1398-1406.

24. Poindexter BB, Feng R, Schmidt B, et al. Comparisons and Limitations of Current Definitions of Bronchopulmonary Dysplasia for the Prematurity and Respiratory Outcomes Program. Ann Am 
Thorac Soc. 2015 Dec;12(12):1822-30.

25. Villamor-Martinez E, Álvarez-Fuente M, Ghazi AMT, et al. Association of Chorioamnionitis With Bronchopulmonary Dysplasia Among Preterm Infants: A Systematic Review, Meta-analysis, and Metaregression. JAMA Netw Open. 2019 11;2(11):e1914611.

26. Pietrasanta C, Pugni L, Merlo D, et al. Impact of different stages of intrauterine inflammation on outcome of preterm neonates: Gestational age-dependent and -independent effect. PLoS One. 2019;14(2):e0211484.

27. Schmidt B, Roberts RS, Davis P, et al. Caffeine therapy for apnea of prematurity. N Engl J Med. 2006 May;354(20):2112-21.

28. Jobe AH. Caffeine therapy-when and why? J Pediatr. 2017 11;190:2.

29. Zysman-Colman Z, Tremblay GM, Bandeali S, et al. Bronchopulmonary dysplasia - trends over three decades. Paediatr Child Health. 2013 Feb;18(2):86-90.

30. Laughon MM, Langer JC, Bose CL, et al. Prediction of bronchopulmonary dysplasia by postnatal age in extremely premature infants. Am J Respir Crit Care Med. 2011 Jun;183(12):1715-22.

31. Naeye RL, Freeman RK, Blanc WA. Nutrition, sex, and fetal lung maturation. Pediatr Res. 1974 Mar;8(3):200-4.

32. Lorente-Pozo S, Parra-Llorca A, Torres B, et al. Influence of Sex on Gestational Complications, Fetalto-Neonatal Transition, and Postnatal Adaptation. Front Pediatr. 2018;6:63.

33. Fulton CT, Cui TX, Goldsmith AM, et al. Gene Expression Signatures Point to a Male Sex-Specific Lung Mesenchymal Cell PDGF Receptor Signaling Defect in Infants Developing Bronchopulmonary Dysplasia. Sci Rep. 2018 11;8(1):17070.

34. Kitterman JA, Chapin CJ, Vanderbilt JN, et al. Effects of oligohydramnios on lung growth and maturation in the fetal rat. Am J Physiol Lung Cell Mol Physiol. 2002 Mar;282(3):L431-9.

35. Lindner W, Pohlandt F, Grab D, et al. Acute respiratory failure and short-term outcome after premature rupture of the membranes and oligohydramnios before 20 weeks of gestation. J Pediatr. 2002 Feb;140(2):177-82.

36. Weiner E, Barrett J, Zaltz A, et al. Amniotic fluid volume at presentation with early preterm prelabor rupture of membranes and association with severe neonatal respiratory morbidity. Ultrasound Obstet Gynecol. 2019 Dec;54(6):767-773.

37. Aikio O, Metsola J, Vuolteenaho R, et al. Transient defect in nitric oxide generation after rupture of fetal membranes and responsiveness to inhaled nitric oxide in very preterm infants with hypoxic respiratory failure. J Pediatr. 2012 Sep;161(3):397-403.e1.

38. Mirza H, Garcia JA, Crawford E, et al. Natural History of Postnatal Cardiopulmonary Adaptation in Infants Born Extremely Preterm and Risk for Death or Bronchopulmonary Dysplasia. J Pediatr. 2018 07;198:187-193.e1.

39. Chandrasekharan P, Kozielski R, Kumar VH, et al. Early Use of Inhaled Nitric Oxide in Preterm Infants: Is there a Rationale for Selective Approach? Am J Perinatol. 2017 04;34(5):428-440. 
40. Kim DH, Kim HS, Choi CW, et al. Risk factors for pulmonary artery hypertension in preterm infants with moderate or severe bronchopulmonary dysplasia. Neonatology. 2012;101(1):40-6.

41. Mourani PM, Abman SH. Pulmonary vascular disease in bronchopulmonary dysplasia: pulmonary hypertension and beyond. Curr Opin Pediatr. 2013 Jun;25(3):329-37.

42. Abman SH. Bronchopulmonary dysplasia: "a vascular hypothesis". Am J Respir Crit Care Med. 2001 Nov;164(10 Pt 1):1755-6.

43. Bhatt AJ, Pryhuber GS, Huyck $H$, et al. Disrupted pulmonary vasculature and decreased vascular endothelial growth factor, Flt-1, and TIE-2 in human infants dying with bronchopulmonary dysplasia. Am J Respir Crit Care Med. 2001 Nov;164(10 Pt 1):1971-80.

44. Check J, Gotteiner N, Liu X, et al. Fetal growth restriction and pulmonary hypertension in premature infants with bronchopulmonary dysplasia. J Perinatol. 2013 Jul;33(7):553-7.

45. Taglauer E, Abman SH, Keller RL. Recent advances in antenatal factors predisposing to bronchopulmonary dysplasia. Semin Perinatol. 2018 Nov;42(7):413-424.

46. Laughon M, Allred EN, Bose C, et al. Patterns of respiratory disease during the first 2 postnatal weeks in extremely premature infants. Pediatrics. 2009 Apr;123(4):1124-31.

\section{Tables}

Due to technical limitations, table 1-4 is only available as a download in the Supplemental Files section.

\section{Figures}

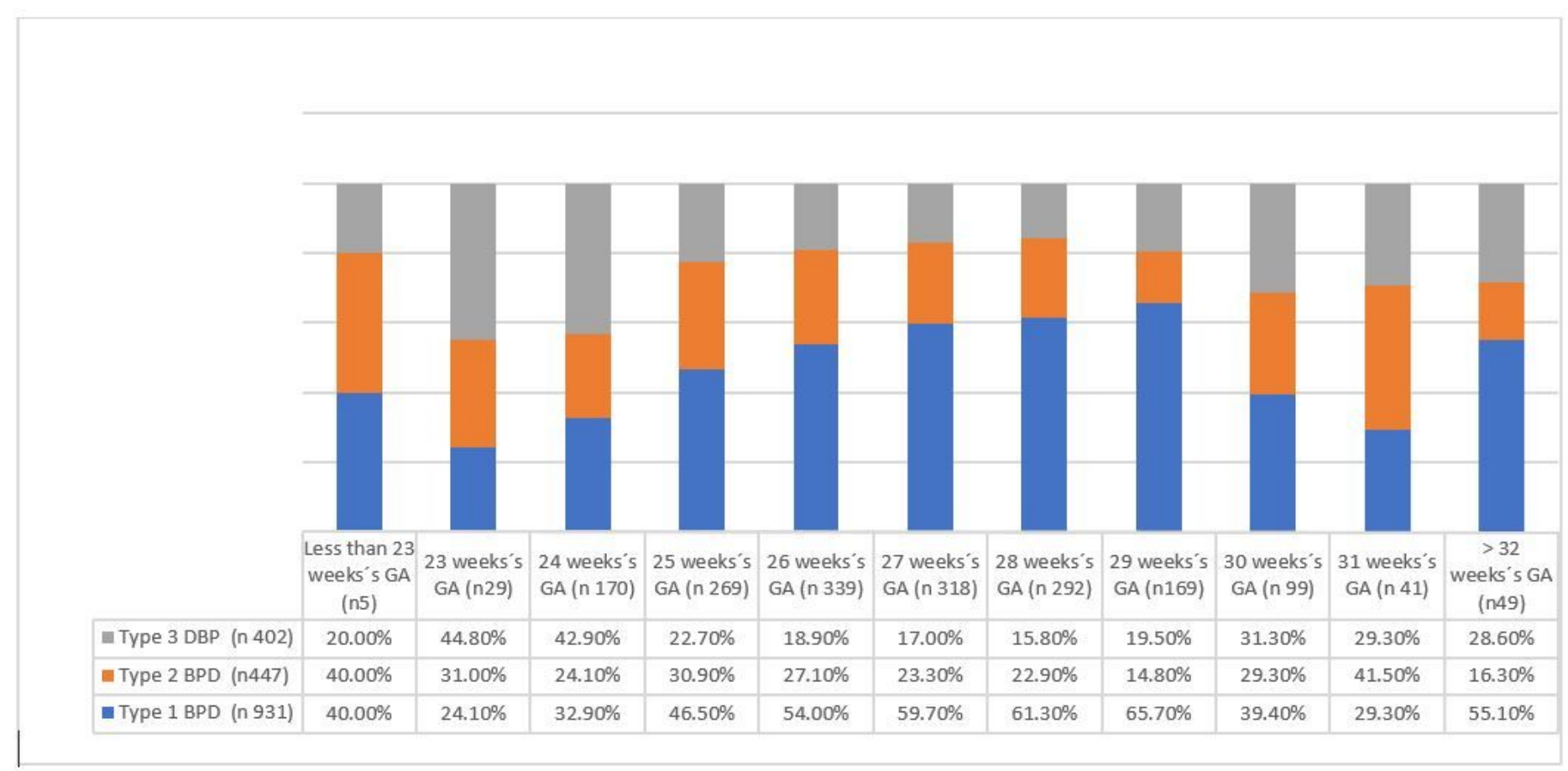


Distribution of types of bronchopulmonary dysplasia according to gestational age at birth in weeks (week 's GA).

\section{Supplementary Files}

This is a list of supplementary files associated with this preprint. Click to download.

- SUPPLEMENTARYINFORMATION1docX.docx

- SuplementarytablesGEIDIS.docx

- TABLES.GEIDIS.docx.docx 